\title{
PHASE SENSITIVE X-RAY IMAGING: TOWARDS ITS INTERDISCIPLINARY APPLICATIONS
}

\author{
C. Kottler ${ }^{\mathrm{a}}$, V. Revol ${ }^{\mathrm{a}, \mathrm{g}}$, R. Kaufmann ${ }^{\mathrm{a}}$, C. Urban ${ }^{\mathrm{a}}$, K. Knop ${ }^{\mathrm{a}}$, U. Sennhauser ${ }^{\mathrm{b}}$,

 \\ E. Knop ${ }^{\text {and N. Blanc }}{ }^{\mathrm{a}}$ \\ ${ }^{a}$ CSEM SA, Photonics Division, Technoparkstrasse 1, CH-8005 Zürich, Switzerland \\ ${ }^{b}$ Laboratory for Electronics/Metrology/Reliability, Empa, Ueberlandstrasse 129, 8600 Duebendorf, Switzerland \\ ${ }^{c}$ CSEM SA, Microsystems Technology, Rue Jaquet-Droz 1, 2002 Neuchâtel, Switzerland \\ ${ }^{d}$ University of Zurich, Institute of Anatomy, Winterthurerstr. 190, CH-8057 Zurich, Switzerland \\ ${ }^{e}$ UniversitätsSpital Zürich, Klinik für Kiefer- und Gesichtschirurgie, Franenklinikstrasse 24, CH-8091 Zürich, \\ Switzerland \\ ${ }^{f}$ University of Bern, Community Ecology, Baltzerstr. 6, CH-3012 Bern, Switzerland \\ ${ }^{g}$ University of Zurich, Institute of Physics, Winterthurerstr. 190, CH-8057 Zurich, Switzerland
}

\begin{abstract}
X-ray phase imaging including phase tomography has been attracting increasing attention during the past few decades. The advantage of X-ray phase imaging is that an extremely high sensitivity is achieved for weakly absorbing materials, such as biological soft tissues, which generate a poor contrast by conventional schemes. Especially for such living samples, where the reduction of the applied dose is of paramount interest, phase sensitive measurements schemes have an inherent potential for a significant dose reduction combined with an image quality enhancement. Several methods have been invented for x-ray phase contrast imaging that either use an approach based on interferometry, diffraction or wave-field propagation. Some of these techniques have a potential for commercial applications, such as in medicine, nondestructive testing, security and inspection. The scope of this manuscript thus deals with one particular such technique that measures the diffraction caused by the specimen by means of a grating interferometer. Examples of measurements are shown that depict the potential of phase contrast imaging for future commercial applications, such as in medical imaging, non-destructive testing and inspection for quality control. The current state of the technology is briefly reviewed as well as its shortcomings to be overcome with regard to the applications.
\end{abstract}

Keywords: X-ray Imaging, Phase Contrast, Interferometry, Computerized Tomography

PACS: $41.50 .+\mathrm{h}, 07.85 .-\mathrm{m}$

\section{INTRODUCTION}

X-ray phase contrast imaging is gaining more and more interest not only in academic research but also for commercial applications. When compared to conventional absorption based radiography, phase sensitive imaging shows substantially increased contrast in weakly absorbing materials, such as plastics or soft tissue. In particular for biological samples, where dose deposition matters, phase contrast techniques are very promising ${ }^{1-5}$. But also in applications for which the radiation dose is not an issue, phase contrast imaging offers the advantage that light elements, e.g. plastics, can be resolved in a dense matrix. Very often, this is a shortcoming of traditional radiography and tomography for instance in non-destructive testing because the strong absorber prevails and the light element's signal vanishes.

Phase contrast imaging has been originally developed mostly on high-brilliance coherent sources, like synchrotron radiation facilities ${ }^{6-14}$. Recently, it was demonstrated that differential phase contrast imaging based on grating interferometry (dPCI) can be successfully implemented on conventional X-ray sources ${ }^{15-18}$. This method has a very high potential in regard to its commercial application essentially because it doesn't have high demands neither on the spatial nor on the temporal coherency of the x-ray source. Indeed, the requirements on coherency are fulfilled 
thanks to an absorbing mask that divides the $\mathrm{x}$-ray beam into several sufficiently coherent, but mutually incoherent line sources. Thereby, the spatial resolution is decoupled from any requirement on coherency. Grating interferometry can be applied in combination with state-of-the-art x-ray equipment (polychromatic high power tubes, large area image sensors), it provides large fields-of-view and, moreover, it is fully compatible with conventional absorption based radiography and tomography schemes. Therefore, dPCI is a promising candidate to become the technology that will bring phase-sensitive X-raying into commercial applications. Since this approach delivers absorption, phase and dark-field signals at the same time, it might be very useful also for applications outside the medical environment, like luggage screening or non-destructive testing.

Today's technology however is not yet ready for a specific commercial application because its industrial and/or medical implementation isn't straight forward, essentially for the following two reasons: First, the extraction and interpretation of the features obtained with the new imaging modality is still unknown. This assessment is clearly a prerequisite for the successful application, especially in medical imaging. Secondly, the targeted applications set very stringent and specific requirements on the instrumentation. These requirements relate in particular to the size of the field of view, the mechanical stability and/or the flexibility, environmental conditions (temperature, vibrations, etc.), measurement time and sample throughput. Furthermore, for the reason of achieving sufficient transmission through the specimen most applications require much higher X-ray energies than what can be reached with today's technology. The higher the X-ray energy the more difficult becomes the fabrication of the diffraction gratings.

\section{INSTRUMENTATION}

The principle of the dPCI method is based on detecting minute changes in the direction of propagation, which are caused by refraction of the X-rays passing through a phase shifting object. Equivalent to refraction in the visible range, the change in direction is proportional to the local gradient in phase shift. However, it should be noted, that the refractive power of matter for X-rays is many orders of magnitude weaker. In FIGURE 1 a schematic overview of the experimental set-up is shown. The essential part of the interferometer consists of two gratings placed between the object and the image detector. While the analyzer grating $\mathrm{G}_{2}$ close to the detector consists of an array of highly absorbing gold lines, the beam splitter grating $G_{1}$ just downstream of the object is made of phase shifting lines. $G_{1}$ creates an interference pattern of very closely spaced lines which cannot be directly resolved by the detector. Therefore the grating $G_{2}$ is necessary, which acts as an array of collimating slits. The total intensity arriving at the detector strongly depends on the relative position of the two gratings and the angle of incidence. Any local refractive index gradient in the object causes a shift of the interference pattern and thus a local change in the intensity recorded on the detector. Note that the described set-up does not require monochromatic radiation and therefore the requirements on temporal coherency are very poor. As mentioned above the requirements for spatial coherency are met by introducing an absorbing mask just downstream of the source $\left(\mathrm{G}_{0}\right.$, source grating).

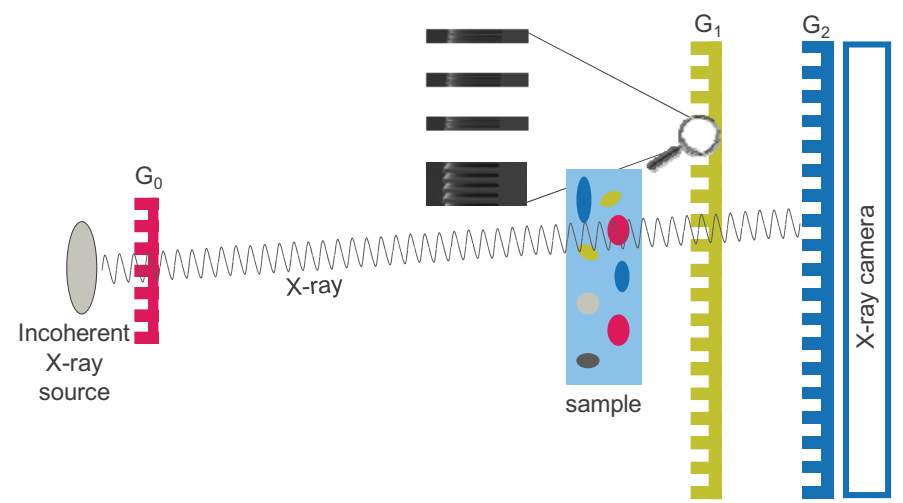

FIGURE 1. Schematic overview of the grating interferometer set-up used for X-ray differential phase contrast imaging (dPCI): It comprises an incoherent $\mathrm{X}$-ray tube source and a digital image recording detector in combination with three X-ray diffraction gratings $\mathrm{G}_{0}$ (source grating), $\mathrm{G}_{1}$ (beam splitter grating) and $\mathrm{G}_{2}$ (analyzer grating).

Our set-up is equipped with a conventional X-ray tube source (Comet MXR-160 HP 20), which has a focal spot size of $1 \times 1 \mathrm{~mm}^{2}($ EN 12543) and a maximum power of $2 \mathrm{~kW}$. We used a digital, scintillator based detector (Rad Icon Shad-o-Box $2 \mathrm{k}$ ) that has $2048 \times 1024$ pixels with a size of $48 \times 48 \mu \mathrm{m}^{2}$, each. The parameters defining the 
interferometer and the gratings were as follows: The periodicities of $G_{0}, G_{1}$ and $G_{2}$ were $p_{0}=57 \mu \mathrm{m}, p_{1}=2.85 \mu \mathrm{m}$ and $\mathrm{p}_{2}=3.0 \mu \mathrm{m}$. The grating's depths were $\mathrm{H}_{0}=60 \mu \mathrm{m}, \mathrm{H}_{1}=12.7 \mu \mathrm{m}$ and $\mathrm{H}_{2}=30 \mu \mathrm{m}$, meaning the structure depth of the Gold bars (for $\mathrm{G}_{0}$ and $\mathrm{G}_{2}$ ) and of the Silicon bars $\left(\mathrm{G}_{1}\right)$. The distances between the gratings were $1=131 \mathrm{~cm}$ between $G_{0}$ and $G_{1}$ and $d=6.9 \mathrm{~cm}$ between $G_{1}$ and $G_{2}$. More details on the set-up can be found in elsewhere ${ }^{19}$.

\section{APPLICATIONS}

In the following section some examples of measurements are presented for samples representative of various application fields. The measurements were performed with the grating interferometer set-up described above. The X-ray tube source was operated at $40 \mathrm{kV}$ and $22.5 \mathrm{~mA}$. The measurement time was typically a few minutes per sample, divided into 20 individual exposures, one for each phase-step. These samples and measurements demonstrate the relevance of $\mathrm{dPCI}$ for a potential implementation in industry and medicine.

As a first example, FIGURE 2 shows the results of the dPCI measurement of a syringe where a needle is glued into a glass-body. The surface of the needle covered by the epoxy glue is critical to ensure that the needle will not be pulled out during its extraction from the patient. For this reason, manufacturers are interested in a non-destructive testing method which allows controlling the glue coverage. As can be seen in the absorption radiograph in FIGURE 2 (A), the glue doesn't give rise to an image contrast because the strong signal of the metal prevails. The differential phase contrast image in (B) however clearly reveals the penetration of the glue along the needle. The linear profiles in (C) taken at different positions (as indicated) reflect the different materials present in the respective circular cross-section. In the cross-section 1, only the glass-body of the syringe is present and the signal shown in the enlarged section results from the interface between the glass body and the air. In cross-section 2, an additional signal can be seen which is due to the second interface between the air and the metal needle. Finally, in cross-section 3 , the glue has replaced the air between the glass body and the needle. The signal from the first interface is indeed significantly lower since the index of refraction of glass is much closer to the one of glue than to the one of air.
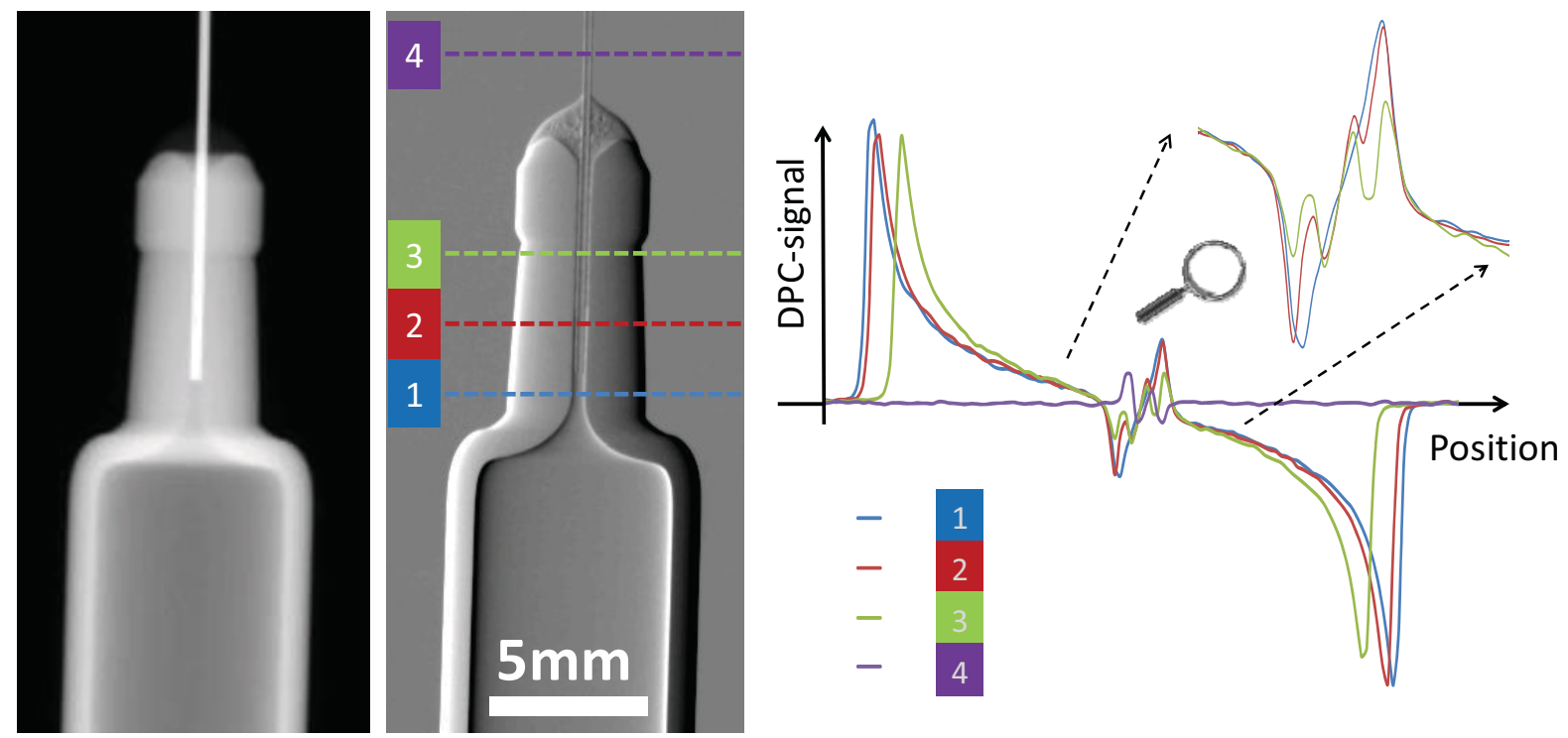

FIGURE 2. (A) and (B) show the absorption radiograph and the differential phase contrast image of a syringe, respectively. The profiles of the differential phase contrast signal in (C) represent vertical positions through the syringe only (1), syringe and needle without (2) and with (3) glue and through the needle only (4).

FIGURE 3 shows the results obtained from the tomographic measurement of a playmobil figurine made of plastic. The absorption radiograph (A), differential phase contrast image (B) and the dark-field image (C) of a single projection are shown. For the tomography 721 exposures in total were taken, distributed over $360^{\circ}$. From the acquired data set the $3 \mathrm{D}$ distribution map of the absorption coefficient and the refraction index were reconstructed ${ }^{20}$. Cross-section along three different directions of the reconstructed volume are shown in (D,E,F). These false color images (mapping of measured coefficients with a color gradient) represent the measured refraction indices and absorption coefficients and thus reveal different types of plastic materials. 

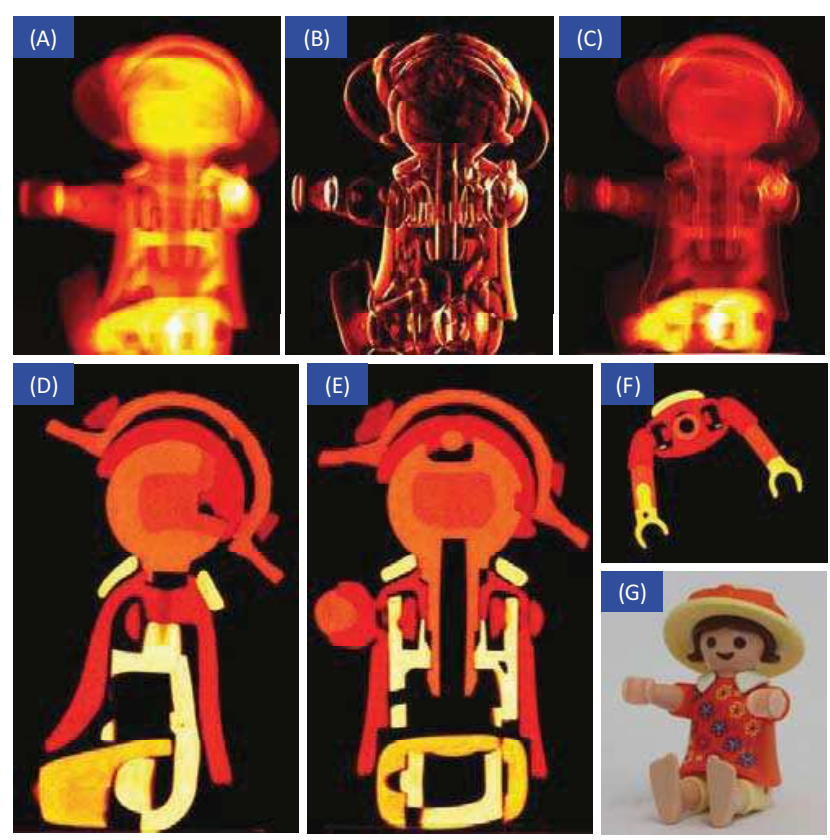

FIGURE 3. Tomography of a playmobil figurine: The images (A, B, C) show the absorption image, the differential phase contrast image and the dark-field image of a single projection measurement of the sample, respectively. Images (D, E, F) are cross-sections along different directions through the figurine obtained from the tomographic reconstruction. A photograph of the figurine is shown in $(\mathrm{G})$.

The two previous examples thus prove that dPCI provides a method to investigate the properties of manufactured objects. Such a technique could be implemented by engineers to control and improve further their fabrication processes. Another type of application that could be envisioned is food control. Food suppliers are indeed confronted with stringent quality controls and have to verify, for example, that no unwished material landed in the products. Absorption based radiography is already implemented and proved useful to detect strong absorbers like metal or rubber pieces. It becomes however useless when the unwished material has a density close to the one of the product, like for instance a piece of fabric from the workers' clothes.

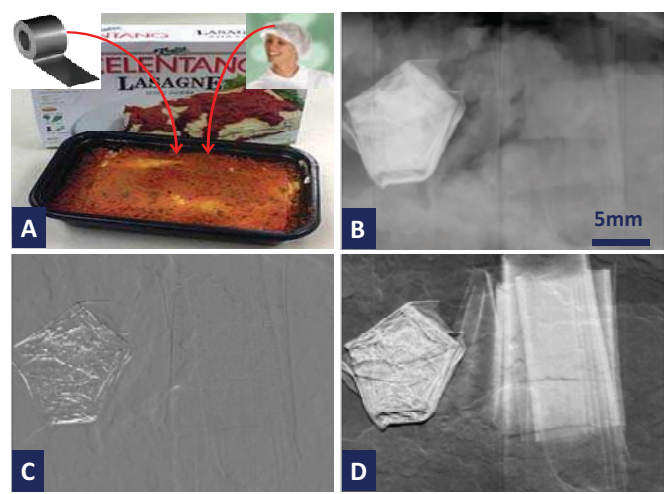

FIGURE 4. The convenience food lasagna was prepared as shown on the photograph in (A) with a plastic tape inside it at one spot (left) and a fabric at a second spot (right). The images (B,C,D) are the absorption image, phase contrast image and the darkfield image, respectively.

FIGURE 4 shows an example measurement of convenience food lasagna which was prepared as shown on the photograph in (A) with a rumpled plastic tape inside it at one spot (left) and a textile fabric at a second spot (right). The plastic tape is visible in the absorption image (B) and can be detected while the fabric is invisible. The latter appears however clearly in the dark field image (D). The textile forms indeed a mesh of fibers which scatters strongly the X-rays and results in a strong signal in the dark field image. 
Finally, medical imaging represents certainly the largest market for dPCI but it is also the most challenging. The change from today's absorption-based radiography to dPCI needs to be motivated by consequent improvements in the detection capabilities. These improvements have to be demonstrated by teams combining experts from the technology and from the medical field.
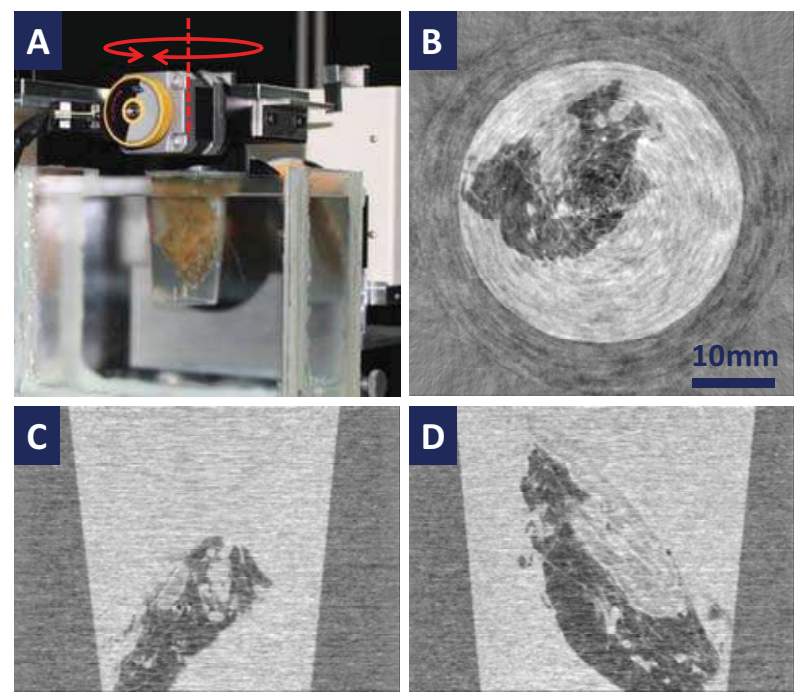

FIGURE 5. (A) Photograph of adrenal gland sample during tomography. Horizontal (B) and vertical (C,D) cross-sections through the reconstructed volume from the differential phase contrast images.

As already stated above, the interpretation of the dPCI images is a major difficulty in medical imaging since clinicians do not have experience with such techniques. To solve this problem, a database of dPCI images needs to be created. It would thus allow the cartography and classification of the human tissues to support the clinicians. For this reason, a study was started recently with the Institute of Anatomy from the University of Zürich in order to produce an atlas of different organs of the human body.

FIGURE 5 illustrates phase contrast tomography applied to a prepared anatomical sample of human adrenal gland. The photograph (A) shows the sample during the tomography: the adrenal gland is embedded and fixed in a cup filled with agarose. For the measurement the cup was turned in a plane-parallel container filled with water (total thickness $\approx 7 \mathrm{~cm})$. In (B) horizontal as well as vertical $(C, D)$ cross-sections through the reconstructed volume from the differential phase contrast images are shown. Inside the cone-shaped cup two different types of the soft tissue are revealed, the adrenal gland (dark region) and the surrounding fat tissue (bright region). During this project, we plan to further improve the image quality, phase sensitivity and acquire data of many other organs as well.

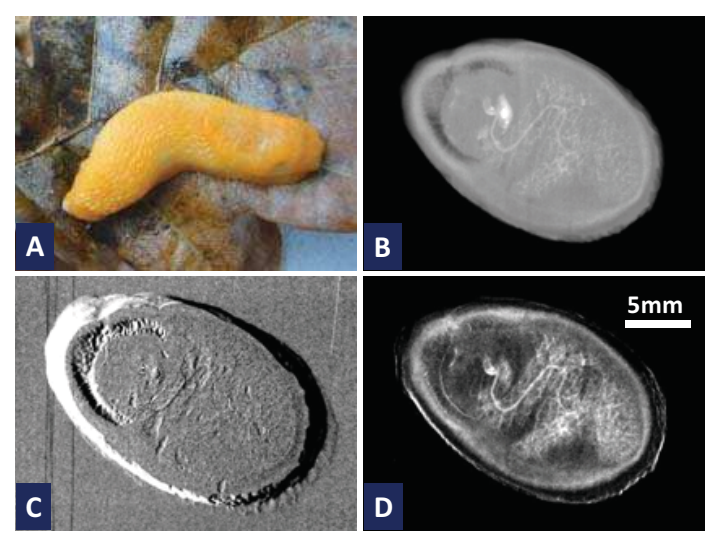

FIGURE 6. Photograph (A) and images from the dPCI measurement of a living snail Arion rufus (Linnaeus 1758): absorption image (B), differential phase contrast image (C) and dark-field image (D). 
Another example closely related to medical imaging is depicted in FIGURE 6. A dPCI measurement of a living snail Arion rufus (Linnaeus 1758) provided by the Department of Community Ecology of the University of Berne was realized. The snail was squeezed between two Plexiglas planes in order to immobilize it and to offer a homogeneously thick cross-section over the area of interest. The photograph (A) shows the Arion rufus after the Xraying, remobilized and in good shape. On the dPCI images (B,C,D), the snail is aligned with its front to the upper left corner of the image and its back in the opposite corner. Though the identification of the organs that are revealed in these images is still ongoing, the winding structure could already be attributed to the intestinal tract.

\section{SUMMARY AND OUTLOOK}

Examples of dPCI measurements were provided above which illustrates the usefulness of dPCI in different fields of applications. dPCI clearly adds to or complements the information revealed by traditional X-raying in specific situations. The strength of phase sensitive X-raying, and of dPCI in particular, resides indeed in the mechanism of image contrast formation that is highly sensitive to poorly absorbing materials, especially when strong absorbers are also present in the specimen.

However, today's technology is not yet ready for commercial implementation. From the instrumentation point of view, one of the most difficult challenges to be met concerns the usable X-ray energies which need to be considerably higher than what can be achieved with the current technology. X-ray gratings are being further developed to reach this goal but the solution may also require the creation of new concepts. Furthermore, other technical solutions will be required that can cope with application specific challenges. As an example, efficient scanning systems have to be developed which allow sufficient sample throughputs in industrial inspection.

Finally, the interpretation of dPCI images needs to be developed by a systematic approach for each field of application. This specifically applies to the biomedical field, where an atlas of the human organs is, for instance, being created that will support clinicians in their analysis. On the other hand, successful applications of dPCI will have to be discovered outside the traditional X-raying fields, where it has not been the method of choice yet.

\section{ACKNOWLEDGMENTS}

We gratefully acknowledge the Division Microsystems Technology from CSEM in Neuchâtel for the gratings' fabrication. This work was supported by the CCMX-NMMC platform (Grant Nr. 0206088).

\section{REFERENCES}

1 A. Momose, T. Takeda, Y. Itai, and K. Hirano, Nature Medicine, 2, 473 (1996).

2 D. Chapman, W. Thomlinson, R. Johnston, D. Washburn, E. Pisano, N. Gmur, Z. Zhong, R. Menk, F. Arfelli, and D. Sayers, Phys. Med. Biol., 42, 2015 (1997).

3 M. Wernick, O. Wirjadi, D. Chapman, Z. Zhong, N. Galatsanos, Y. Yang, J. Brankov, O. Oltulu, M. Anastasio, and C. Muehleman, Phys. Med. Biol., 48, 3875 (2003).

4 A. Momose, Opt. Express 11, 2303-2314 (2003)

5 M. Bech, T. H. Jensen, O. Bunk, T. Donath, C. David, T. Weitkamp, G. LeDuc, A. Bravin, P. Cloetens, F. Pfeiffer, Z. Med. Phys., in press., (2010).

6 R. Fitzgerald, Phys. Today, 53, 23 (2000).

7 U. Bonse, M. Hart, Appl. Phys. Lett., 6, 155 (1965).

8 V.V. Protopopov, and J. Sobota, ” Opt. Commun. 213, 267-279 (2002).

9 V. N. Ingal and E. A. Beliaevskaya, J. Phys. D 28, 2314-2317 (1995).

10 T. J. Davis, D. Gao, T. E. Gureyev, A. W. Stevenson, and S. W. Wilkins, Nature (London) 373, 595-598 (1995).

11 S. Wilkins, T. Gureyev, D. Gao, A. Pogany, and A. Stevenson, Nature 384, 335 (1996).

12 A. Olivo and R. Speller, Phys. Med. Biol., 52, 6555 (2007).

13 Z-T Wang, K-J Kang, Z-F Huang and Z-Q Chen, Appl. Phys. Letters 95, 094105 (2009).

14 T. Weitkamp, A. Diaz, C. David, F. Pfeiffer, M. Stampanoni, P. Cloetens, and E. Ziegler, Opt. Express 13, 6396 (2005).

15 F. Pfeiffer, M. Bech, O. Bunk, P. Kraft, E. Eikenberry, C. Brönnimann, C. Grünzweig, and C. David, Nature Materials 7, 134 (2008).

16 F. Pfeiffer, T. Weitkamp, O. Bunk, and C. David, Nature Phys. 2, 258 (2006).

17 C. David, F. Pfeiffer, and T. Weitkamp, Patent \# EP0511920060530 (2006).

18 C. Kottler, F. Pfeiffer, O. Bunk, C. Grünzweig, and C. David, Rev. Sci. Instrum. 78, 043710 (2007).

19 V. Revol, C. Kottler, R. Kaufmann, U. Straumann and C. Urban, in preparation (2010).

20 F. Pfeiffer, C. Kottler, O. Bunk, and C. David, Phys. Rev. Lett. 98, 108105/1 (2007). 